\title{
Generalization of Some Problems with $s$-Separation
}

\author{
Beih El-Sayed El-Desouky, Mohamed Moustafa Gad, Shimaa El-Eraqy \\ Department of Mathematics, Faculty of Science, Mansoura University, Mansoura, Egypt \\ Email: b_desouky@yahoo.com
}

Received 29 October 2014; revised 26 November 2014; accepted 18 December 2014

Copyright (C) 2015 by authors and Scientific Research Publishing Inc.

This work is licensed under the Creative Commons Attribution International License (CC BY).

http://creativecommons.org/licenses/by/4.0/

\section{(c) (†) Open Access}

\begin{abstract}
In this article we apply and discuss El-Desouky technique to derive a generalization of the problem of selecting $\boldsymbol{k}$ balls from an $\boldsymbol{n}$-line with no two adjacent balls being $\boldsymbol{s}$-separation. We solve the problem in which the separation of the adjacent elements is not having odd and even separation. Also we enumerate the number of ways of selecting $k$ objects from $n$-line objects with no two adjacent being of separations $m, m+1, \cdots, p m$, where $p$ is positive integer. Moreover we discuss some applications on these problems.
\end{abstract}

\section{Keywords}

Probability Function, $s$-Separation, $s$-Successions, $n$-Line, $n$-Circle

\section{Introduction}

Kaplansky [1] (see also Riordan ([2] p. 198, lemma) and Moser [3]) studied the problem of selecting $k$ objects from $n$ objects arranged in a line (called $n$-line) or a circle (called $n$-circle) with no two selected objects being consecutive. Let $f(x, y)$ and $g(x, y)$ denote the number of ways of such selections for $n$-line and $n$-circle respectively. Kaplansky proved that

$$
f(n, k)= \begin{cases}\left(\begin{array}{c}
n-k+1 \\
k
\end{array}\right), & 0 \leq k \leq n \\
0, & \text { otherwise, }\end{cases}
$$

and

$$
g(n, k)= \begin{cases}\frac{n}{k}\left(\begin{array}{c}
n-k-1 \\
k-1
\end{array}\right), & n \geq 2 k+1 \\
0, & 1 \leq n \leq k .\end{cases}
$$


El-Desouky [4] studied another related problem with different techniques and proved that

$$
l(n, k)= \begin{cases}\sum_{i=0}^{\lambda}\left(\begin{array}{c}
k-1 \\
i
\end{array}\right)\left(\begin{array}{c}
n-k+1-i \\
i+1
\end{array}\right), \quad \lambda=\min \left(k-1,\left[\frac{n-k}{2}\right]\right), 0 \leq k \leq n \\
0, & \text { otherwise, }\end{cases}
$$

where $l(n, k)$ is the number of ways of selecting $k$ balls from $n$ balls arranged in a line with no two adjacent balls being unit separation.

In the following we adopt some conventions: $\left[x^{n}\right] f(x)$ denotes the coefficient of $x^{n}$ in the formal power series $f(x) ;\left[x^{n} y^{m}\right] f(x, y)$ denotes the coefficient of $x^{n} y^{m}$ in the series $f(x, y) ;[x]$ is the largest integer less than or equal to $x, N=\{0,1, \cdots\}$ and $N_{n}=\{1,2,3, \cdots\}$.

Also, El-Desouky [5] derived a generalization of the problem given in [4] as follows: let $l_{s}(n, k)$ denote the number of ways of selecting $k$ balls from $n$ balls arranged in a line with no two adjacent balls from the $k$ selected balls being $s$-separation; two balls have separation $s$ if they are separated by exactly $s$ balls. Let $d_{s}(n, k)$ denote the number of ways of selecting $k$ balls from $n$ balls arranged in a circle with no two adjacent balls from the $k$ selected balls being s-separation

Let $l_{s}(n, k)$ be as defined before. Then $l_{s}(n, k)$ is equal to the number of $k$-subsets of $N_{n}$ where the difference $s+1$ is not allowed, so

$$
\begin{aligned}
& l_{s}(n, k)=\sum_{i=0}^{v}(-1)^{i}\left(\begin{array}{c}
k-1 \\
i
\end{array}\right)\left(\begin{array}{c}
n-(s+1) i \\
k-i
\end{array}\right) \\
& \text { where } v=\min \left(k-1,\left[\frac{n-k}{s}\right]\right), 0 \leq k \leq n \text {, and } s=0,1, \cdots, n-k \text {. }
\end{aligned}
$$

Let $d_{s}(n, k)$ be as defined before. Then the difference $s+1$ is not allowed, so

$$
\begin{aligned}
& d_{s}(n, k)=\frac{n}{k} \sum_{i=0}^{\beta}(-1)^{i}\left(\begin{array}{c}
k \\
i
\end{array}\right)\left(\begin{array}{c}
n-(s+1) i-1 \\
k-i-1
\end{array}\right), \\
& \text { where } \beta=\min \left(k,\left[\frac{n-k}{s}\right]\right), 0 \leq k \leq n, \text { and } s=0,1, \cdots, n-k .
\end{aligned}
$$

Let $l_{s}(n, k, m)$ be the number of ways of selecting $k$ balls from $n$ balls arranged in a line with exactly $m$ adjacent balls being of separation $s$ or ( $s$-successions), which gives a generalization of (4.1) in El-Desouky [4].

Thus,

$$
\begin{aligned}
& l_{s}(n, k, m)=\sum_{i=m}^{\mu^{\prime}} \sum_{j=0}^{k-1-i}(-1)^{i}\left(\begin{array}{c}
k-1 \\
i
\end{array}\right)\left(\begin{array}{c}
k-1-i \\
j
\end{array}\right)\left(\begin{array}{c}
n-(s+1) i-s j \\
k-m
\end{array}\right), \\
& \text { where } \mu^{\prime}=\min \left(k-1,\left[\frac{n-k+m}{s+1}\right]\right), m=0,1, \cdots, k-1, s=0,1, \cdots, n-k .
\end{aligned}
$$

For more details on such problems, see [3] [6] [7].

\section{Main Results}

We use El-Desouky technique to solve two problems in the linear case, with new restrictions. That is if the separation of any two adjacent elements from the $k$ selected elements being of odd separation and of even separation. Moreover, we enumerate $M_{s}(n, k ; m, p m)$ which denotes the number of ways of selecting $k$ objects from $n$ objects arrayed in a line where any two adjacent objects from the $k$ selected objects are not being of $m, m+$ $1, \cdots, p m$ separations, where $p$ is positive integer.

\subsection{No Two Adjacent Being Odd Separation}

Let $y_{o}(n, k)$ denote the number of ways of selecting $k$ balls from $n$ balls arranged in a line, where the separa- 
tion of any two adjacent balls from the $k$ selected balls being of odd separation. say s, i.e. $s=1,3,5, \cdots$. This means that no two adjacent being of 2, 4, 6, $\cdots$ differences, see Table 1.

So, following Decomposition (2.3.14) see [8] (p. 55), $y_{o}(n, k)$ is equal to the number of $k$-subsets of $N_{n}$ where the differences $s+1, s=1,3,5, \cdots$ are not allowed, hence $y_{o}(n, k)=\left[x^{n}\right] f(x)$, where

$$
\begin{aligned}
f(x) & =\left(x+x^{2}+\cdots\right)\left[x+x^{2}+\cdots-\left(x^{2}+x^{4}+\cdots\right)\right]^{k-1}(1+x+\cdots) \\
& =\frac{x}{1-x}\left[\frac{x}{1-x}-\left(x^{2}+x^{4}+\cdots\right)\right]^{k-1} \frac{1}{1-x} \\
& =\frac{x}{(1-x)^{2}} \frac{x^{k-1}}{(1-x)^{k-1}}\left[1-(1-x)\left(x+x^{3}+\cdots\right)\right]^{k-1} \\
& =x^{k}(1-x)^{-(k+1)}(1-x)^{-(k+1)}
\end{aligned}
$$

hence

$$
f(x)=\sum_{i=0}^{\infty} \sum_{j=0}^{\infty}(-1)^{j} x^{k}\left(\begin{array}{c}
k+i \\
i
\end{array}\right) x^{i}\left(\begin{array}{c}
k+j-2 \\
j
\end{array}\right) x^{j}=\sum_{i=0}^{\infty} \sum_{j=0}^{\infty}(-1)^{j}\left(\begin{array}{c}
k+i \\
i
\end{array}\right)\left(\begin{array}{c}
k+j-2 \\
j
\end{array}\right) x^{i+j+k} .
$$

Setting $n=i+j+k \quad j=n-i-k$ we have

$$
f(x)=\sum_{n=k}^{\infty} \sum_{i=0}^{n-k}(-1)^{n-i-k}\left(\begin{array}{c}
k+i \\
i
\end{array}\right)\left(\begin{array}{c}
k+n-i-k-2 \\
n-i-k
\end{array}\right) x^{n}=\sum_{n=k}^{\infty} \sum_{i=0}^{n-k}(-1)^{n-i-k}\left(\begin{array}{c}
k+i \\
i
\end{array}\right)\left(\begin{array}{c}
n-i-2 \\
n-i-k
\end{array}\right) x^{n} .
$$

Therefore, the coefficient of $x^{n}$ gives

$$
y_{o}(n, k)=\sum_{i=0}^{n-k}(-1)^{n-i-k}\left(\begin{array}{c}
k+i \\
i
\end{array}\right)\left(\begin{array}{c}
n-i-2 \\
k-2
\end{array}\right)
$$

A calculated table for the values of $y_{o}(n, k)$ is given in Table 1 , where $1 \leq n, k \leq 10$.

Remark 1. It is easy to conclude that $y_{o}(n, k)$ satisfies the following recurrence relation

$$
y_{o}(n, k)=y_{o}(n-1, k-1)+y_{o}(n-2, k), \quad n, k \geq 2 \text { and } y_{o}(n, k)=0 \text { for } k>n
$$

with the convention $y_{o}(n, 1)=n, n \geq 1$.

Table 1. A calculated table for the values of $y_{o}(n, k)$.

\begin{tabular}{ccccccccccccc}
\hline$n$ & $k$ & 1 & 2 & 3 & 4 & 5 & 6 & 7 & 8 & 9 & 10 \\
\hline 1 & 1 & 0 & 0 & 0 & 0 & 0 & 0 & 0 & 0 & 0 \\
2 & 2 & 1 & 0 & 0 & 0 & 0 & 0 & 0 & 0 & 0 \\
3 & 3 & 2 & 1 & 0 & 0 & 0 & 0 & 0 & 0 & 0 \\
4 & 4 & 4 & 2 & 1 & 0 & 0 & 0 & 0 & 0 & 0 \\
5 & 5 & 6 & 5 & 2 & 1 & 0 & 0 & 0 & 0 & 0 \\
6 & 6 & 9 & 8 & 6 & 2 & 1 & 0 & 0 & 0 & 0 \\
7 & 7 & 12 & 14 & 10 & 7 & 2 & 1 & 0 & 0 & 0 \\
8 & 8 & 16 & 20 & 20 & 12 & 8 & 2 & 1 & 0 & 0 \\
10 & 9 & 20 & 30 & 30 & 27 & 14 & 9 & 2 & 1 & 0 \\
\hline
\end{tabular}




\subsection{No Two Adjacent Being Even Separation}

Let $y_{e}(n, k)$ denote the number of ways of selecting $k$ balls from $n$ balls arranged in a line, where the separation of any two adjacent balls from the $k$ selected balls are not being of even separation, say si.e. $s=0,2,4, \cdots$. This means that no two adjacent being of $1,3,5, \cdots$ differences.

So, following Decomposition (2.3.14) see [8] (p. 55) then $y_{e}(n, k)$ is equal to the number of $k$-subsets of $N_{n}$ where the differences $s+1, s=0,2,4, \cdots$ are not allowed, hence $y_{e}(n, k)=\left[x^{n}\right] f(x)$, where

$$
\begin{aligned}
f(x) & =\left(x+x^{2}+\cdots\right)\left[x+x^{2}+\cdots-\left(x+x^{3}+\cdots\right)\right]^{k-1}(1+x+\cdots) \\
& =\frac{x}{1-x}\left[\frac{x}{1-x}-\left(x+x^{3}+\cdots\right)\right]^{k-1} \frac{1}{1-x} \\
& =\frac{x}{(1-x)^{2}} \frac{x^{k-1}}{(1-x)^{k-1}}\left[1-(1-x)\left(1+x^{2}+\cdots\right)\right]^{k-1} \\
& =\frac{x^{2 k-1}}{(1-x)^{k+1}(1+x)^{k-1}} \\
& =x^{2 k-1}(1-x)^{-(k-1)}(1+x)^{-(k-1)},
\end{aligned}
$$

hence

$$
f(x)=\sum_{i=0}^{\infty} \sum_{j=0}^{\infty}(-1)^{j} x^{2 k-1}\left(\begin{array}{c}
k+i \\
i
\end{array}\right) x^{i}\left(\begin{array}{c}
k+j-2 \\
j
\end{array}\right) x^{j}=\sum_{i=0}^{\infty} \sum_{j=0}^{\infty}(-1)^{j}\left(\begin{array}{c}
k+i \\
i
\end{array}\right)\left(\begin{array}{c}
k+j-2 \\
j
\end{array}\right) x^{2 k-1+i+j} .
$$

Setting $n=2 k-1+i+j, \quad j=n-2 k+1-i$ we get

$$
f(x)=\sum_{j=0}^{\infty} \sum_{i=0}^{n-2 k+1}(-1)^{n+1-i}\left(\begin{array}{c}
k+i \\
i
\end{array}\right)\left(\begin{array}{c}
k+n-2 k+1-i-2 \\
n-2 k+1-i
\end{array}\right) x^{n}=\sum_{j=0}^{\infty} \sum_{i=0}^{n-2 k+1}(-1)^{n+1-i}\left(\begin{array}{c}
k+i \\
i
\end{array}\right)\left(\begin{array}{c}
n-k-i-1 \\
k-2
\end{array}\right) x^{n} .
$$

Therefore, the coefficient of $x^{n}$ gives

$$
y_{e}(n, k)=\sum_{i=0}^{n-2 k+1}(-1)^{n+1-i}\left(\begin{array}{c}
k+i \\
i
\end{array}\right)\left(\begin{array}{c}
n-k-i-1 \\
k-2
\end{array}\right) .
$$

Moreover in the next subsection, we use our technique to enumerate $M_{s}(n, k ; m, p m)$ the number of ways of selecting $k$ objects from $n$ objects arrayed in a line such that no two adjacent elements have the differences $m+1$, $m+2, \cdots, p m+1$ i.e. no two adjacent element being of $m, m+1, \cdots, p m$ separations, where $p$ is positive integer.

\subsection{Explicit Formula for $M_{s}(n, k ; m, p m)$}

Let $M_{s}(n, k ; m, p m)$ be the number of ways of selecting $k$ objects from $n$ objects arrayed in a line where any two adjacent objects from the $k$ selected objects are not being of $m, m+1, \cdots, p m$ separations, where $p$ is positive integer, hence $M_{s}(n, k ; m, p m)=\left[x^{n}\right] f(x)$, where

$$
\begin{aligned}
f(x) & =\left(x+x^{2}+\cdots\right)\left[x+x^{2}+\cdots-\left(x^{m+1}+x^{m+2}+\cdots+x^{p m+1}\right)\right]^{k-1} \frac{1}{1-x} \\
& =\frac{x^{k}}{(1-x)^{2}}\left[\frac{1-x^{m}}{1-x}+x^{p m+1}(1-x)^{-1}\right]^{k-1} \\
& =x^{k}(1-x)^{-(k+1)}\left[\begin{array}{c}
\left.1-x^{m}\left(1-x^{p m-m+1}\right)\right]^{k-1} \\
\end{array}=\sum_{i=0}^{\infty} \sum_{j=0}^{\infty} \sum_{l=0}^{\infty}(-1)^{i+j}\left(\begin{array}{c}
k-1 \\
i
\end{array}\right)\left(\begin{array}{c}
i \\
j
\end{array}\right) x^{j(p m-m+1)+m i} x^{l}\left(\begin{array}{c}
k+l \\
l
\end{array}\right) x^{k} .\right.
\end{aligned}
$$


Setting $n=j(p m-m+1)+m i+l+k$ it is easy to find the coefficient of $x^{n}$ hence

$$
M_{s}(n, k ; m, p m)=\sum_{i=0}^{k-1} \sum_{j=0}^{i}(-1)^{i+j}\left(\begin{array}{c}
k-1 \\
i
\end{array}\right)\left(\begin{array}{l}
i \\
j
\end{array}\right)\left(\begin{array}{c}
n-j(p m-m+1)-m i \\
k
\end{array}\right) \text {. }
$$

\section{Some Applications}

Let $n$ urns be set out along a line, that is, one-dimensional.

Suppose we have $m$ balls of which $m_{i}$ are of colour $c_{i}, i=1,2, \cdots, k$ and we assign these balls to urns so that, see Pease [9]:

i) No urn contains more than one ball.

ii) All $m_{i}$ balls of colour $c_{i}$ are in consecutive urns, $i=1,2, \cdots, k$.

El-Desouky proved that if the order of colours of the groups is specified, the number of arrangement is just $\left(\begin{array}{c}n-m+k \\ k\end{array}\right)$. Hence if the total number of balls $\sum_{i=1}^{k} m_{i}=2 k-1$, the number of arrangements is $l_{o}(n, k)=f(n, k)=\left(\begin{array}{c}n-k+1 \\ k\end{array}\right)$ as a special case of El-Desouky results [5].

It is of practical interest to find the asymptotic behavior of $f(n, k)$ or the probability $p(n, k)=f(n, k) /\left(\begin{array}{l}n \\ k\end{array}\right)$ for large $n$ and $k$.

Let $X$ be a random variable having the probability function $p(n, k)$ then

$$
P(X=k)=p(n, k)=\frac{\left(\begin{array}{c}
n-k+1 \\
k
\end{array}\right)}{\left(\begin{array}{l}
n \\
k
\end{array}\right)},
$$

so

$$
\begin{aligned}
\ln P(X=k) & =\ln \left[\left(1-\frac{k-1}{n}\right)\left(1-\frac{k}{n}\right) \cdots\left(1-\frac{2(k-1)}{n}\right)\right]-\ln \left[\left(1-\frac{1}{n}\right)\left(1-\frac{2}{n}\right) \cdots\left(1-\frac{k-2}{n}\right)\left(1-\frac{k-1}{n}\right)\right] \\
& \simeq\left[-\frac{k-1}{n}-\frac{k}{n}-\cdots-\frac{2(k-1)}{n}\right]-\left[-\frac{1}{n}-\frac{2}{n}-\cdots-\frac{k-2}{n}-\frac{k-1}{n}\right] \\
& =-\frac{3 k(k-1)}{2 n}+\frac{k(k-1)}{2 n}=-\frac{k(k-1)}{n}
\end{aligned}
$$

where we used the first aproximation

$$
\ln (1-x)=-x
$$

Therefore,

$$
P(X=k)=\mathrm{e}^{-\frac{k(k-1)}{n}} .
$$

Putting $Y=\frac{X}{\sqrt{n}}$ we have

$$
\begin{aligned}
P(Y=t) & =P\left(\frac{X}{\sqrt{n}}=t\right)=P(X=\sqrt{n} t) \\
& =\mathrm{e}^{\frac{-\sqrt{n} t(\sqrt{n} t-1)}{n}}, \text { hence }
\end{aligned}
$$


$\lim _{n \rightarrow \infty} P(Y=t)=\mathrm{e}^{-t^{2}}$

Maosen [10] considered the following problem. Let $t$ be any nonnegative integer.

If we want to select $k$ balls from an $n$-line or an $n$-circle under the restriction that any two adjacent selected balls are not $t$-separated, how many ways are there to do it? He solved these problems by means of a direct structural analysis. For the two kinds of problems, he used $F_{t}(n, k)$ to denote the number of ways of selecting $k$ balls from $n$ balls arranged in a line with no two adjacent selected balls being $t$-separation and $G_{t}(n, k)$ to denote the number of ways of selecting $k$ balls from an $n$-circle with no two adjacent selected being $t$-separation. He proved that

$$
\begin{gathered}
F_{t}(n, k)=\sum_{t \geq 0}(-1)^{t}\left(\begin{array}{c}
k-1 \\
l
\end{array}\right)\left(\begin{array}{c}
n-l(t+1) \\
k-1
\end{array}\right), \\
G_{t}(n, k)=\frac{n}{k}\left\{(-1)^{j}\left(\begin{array}{c}
k \\
j
\end{array}\right)\left(\begin{array}{c}
n-j(t+1)-1 \\
k-1-j
\end{array}\right)+(-1)^{k} \delta[n, k(1+t)]\right\} .
\end{gathered}
$$

Remark 2. In fact El-Desouky [5] has proved (3.2) in 1988.

\section{References}

[1] Kplansky, I. (1943) Solution of the "Problems des Ménages”. Bulletin of the American Mathematical Society, 49, 784-785. http://dx.doi.org/10.1090/S0002-9904-1943-08035-4

[2] Riordan, J. (1958) An Introduction to Combinatorial Analysis. Wiley, New York.

[3] Moser, W.O.J. (1986) The Number of Subsets without a Fixed Circular Distance. Journal of Combinatorial Theory, Series A, 43, 130-132. http://dx.doi.org/10.1016/0097-3165(86)90030-0

[4] El-Desouky, B.S. (1988) On Selecting $k$ Balls from an $n$-Line without Unit Separation. Indian Journal of Pure and Applied Mathematics, 19, 145-148.

[5] El-Desouky, B.S. (1988) Selecting $k$ Balls without s-Separation. The 23rd Annual Conference on Statistics, Computer Science, Operations Research and Mathematics, Cairo, December 1988, 40-46.

[6] Mansour, T. and Sun, Y.D. (2008) On Selecting the Number of Combinations without Certain Separations. European Journal of Combinatorics, 29, 1200-1206. http://dx.doi.org/10.1016/j.ejc.2007.06.024

[7] Mansour, T. (2014) Set Partitions with Circular Successions. European Journal of Combinatorics, 41, $207-216$. http://dx.doi.org/10.1016/j.ejc.2014.06.008

[8] Gourden, J.P. and Jackson, D.M. (1993) Combinatorial Enumeration. Wiley, New York.

[9] Pease, R.W. (1975) General Solution to the Occupancy Problem with Variably Sized Runs of Adjacent Cells Occupied by Single Balls. Mathematics Magazine, 48, 131-134. http://dx.doi.org/10.2307/2689693

[10] Maosen, J. (1995) On Selecting $k$ Balls from an $n$-Line or $n$-Circle without $t$-Separations. Northeastern Mathematical Journal, 11, 355-364. 
Scientific Research Publishing (SCIRP) is one of the largest Open Access journal publishers. It is currently publishing more than 200 open access, online, peer-reviewed journals covering a wide range of academic disciplines. SCIRP serves the worldwide academic communities and contributes to the progress and application of science with its publication.

Other selected journals from SCIRP are listed as below. Submit your manuscript to us via either submit@scirp.org or Online Submission Portal.
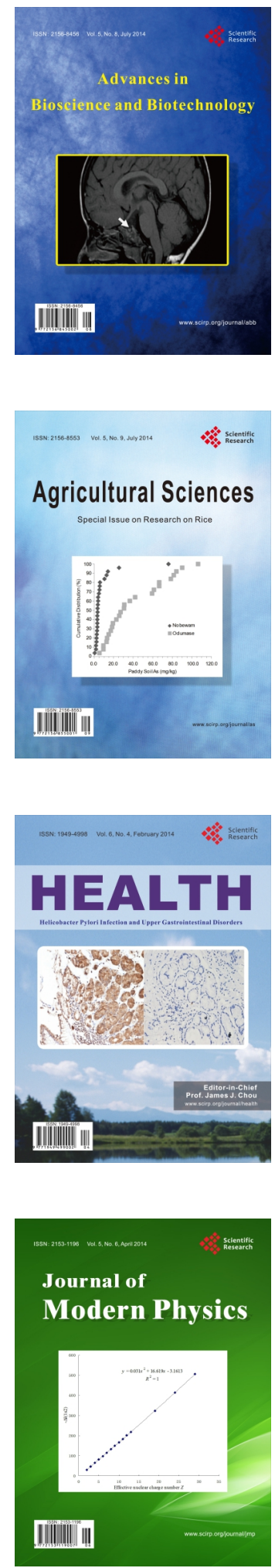
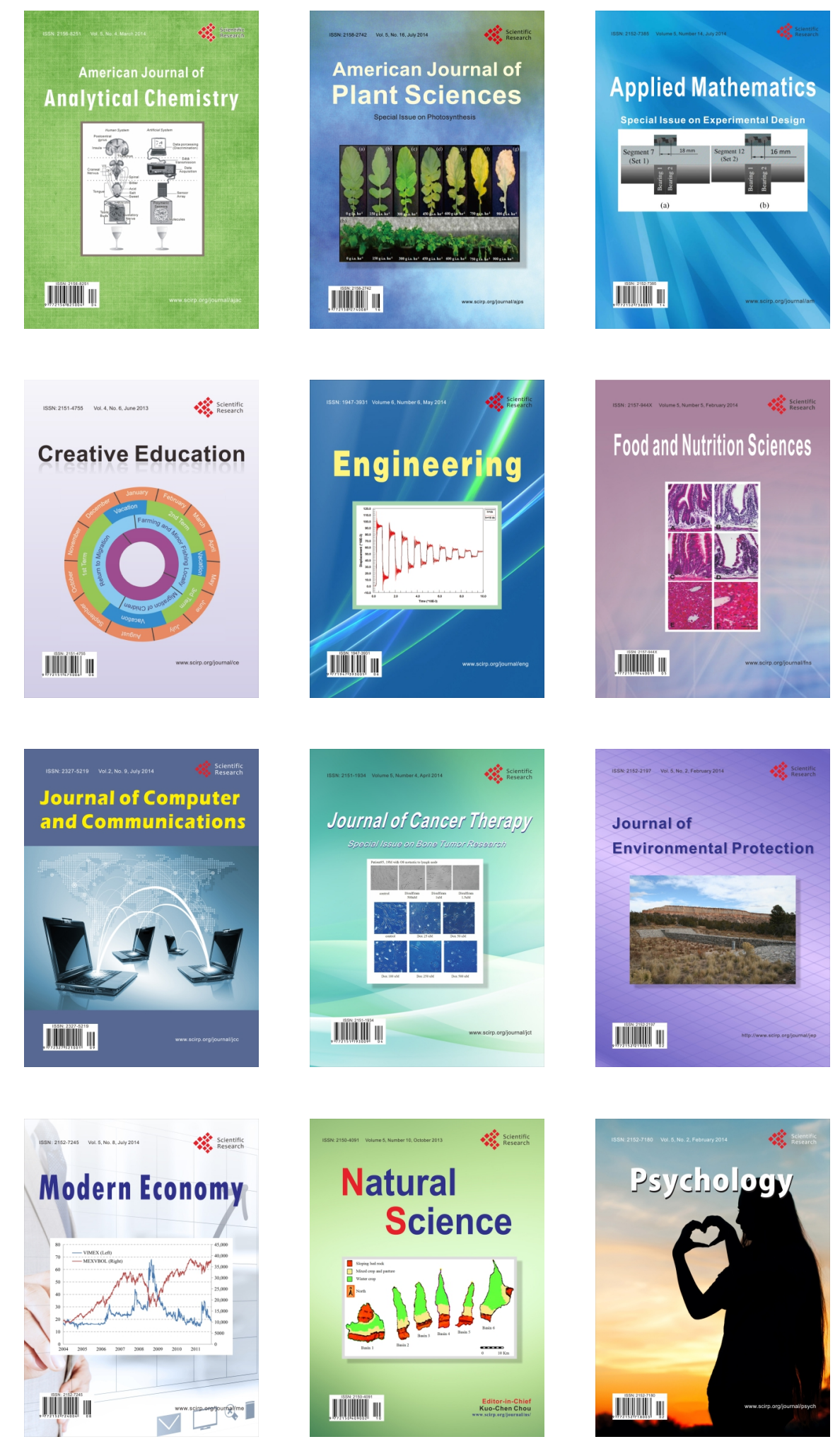